OPEN ACCESS

Edited by:

Pietro Avanzini,

University of Parma, Italy

Reviewed by:

Sandro Legey,

Universidade Veiga de Almeida, Brazil

Federica Sancassiani,

University of Cagliari, Italy

*Correspondence:

Petra Jansen

petra.jansen@psk.uni-regensburg.de

Specialty section: This article was submitted to Movement Science and Sport

Psychology,

a section of the journal

Frontiers in Psychology

Received: 24 July 2018 Accepted: 29 April 2019 Published: 16 May 2019

Citation:

Machado S, Jansen P, Almeida V and Veldema J (2019) Is tDCS an Adjunct

Ergogenic Resource for Improving

Muscular Strength and Endurance

Performance? A Systematic Review.

Front. Psychol. 10:1127.

doi: 10.3389/fpsyg.2019.01127

\section{Is tDCS an Adjunct Ergogenic Resource for Improving Muscular Strength and Endurance Performance? A Systematic Review}

\author{
Sergio Machado ${ }^{1}$, Petra Jansen ${ }^{2 *}$, Victor Almeida ${ }^{1}$ and Jitka Veldema ${ }^{2,3}$ \\ ${ }^{1}$ Laboratory of Physical Activity Neuroscience, Physical Activity Sciences Postgraduate Program, Salgado de Oliveira \\ University (UNIVERSO), Niterói, Brazil, ${ }^{2}$ Faculty of Psychology, Education and Sport Science, University of Regensburg, \\ Regensburg, Germany, ${ }^{3}$ Helios Klinik Kipfenberg, Kipfenberg, Germany
}

Exercise performance is influenced by many physical factors, such as muscle strength and endurance. Particularly in the physical fitness and sports performance contexts, there are many types of ergogenic aids to improve muscular strength and endurance performance, with non-athletes and even athletes using illegal drugs to reach the top. Thus, the development of innovative methods to aid in exercise performance is of great interest. One such method is transcranial direct current stimulation (tDCS). A systematic search was performed on the following databases, until January 2019; PubMed/MEDLINE, SCOPUS, and Pedro database. Studies on tDCS for muscular strength and endurance performance improvement in non-athletes and athletes adults were included. We compared the effect of anodal-tDCS (a-tDCS) to a sham/control condition on the outcomes muscular strength and endurance performance. We found 26 controlled trials. No trial mentions negative side effects of the intervention. The data show differences between the studies investigating muscle strength and the studies evaluating endurance, with regard to successful use of tDCS. Studies investigating the efficiency of tDCS on improving muscular strength demonstrate positive effects of a-tDCS in $66.7 \%$ of parameters tested. In contrast, in studies evaluating the effects of a-tDCS on improving endurance performance the a-tDCS revealed a significant improvement in only $50 \%$ of parameters assessed. The majority of the data shows consistently influence of a-tDCS on muscular strength, but not to endurance performance. The results of this systematic review suggest that a-tDCS can improve muscular strength, but not to endurance performance.

Keywords: transcranial direct current stimulation, tDCS, muscular strength, endurance, systematic review

\section{INTRODUCTION}

Exercise performance is influenced by many physical factors, such as muscle strength and endurance (Sleivert and Rowlands, 1996; Neumayr et al., 2003; McCormick et al., 2015). Particularly in the physical fitness and sports performance contexts, there are many types of ergogenic aids to improve muscular strength and endurance performance (Schubert and Astorino, 2012), with non-athletes and even athletes using illegal drugs to reach the top (Savulescu et al., 2004). Some 
years ago, sport scientists started to focus on the study of the brain as the central governor, and thus, regulates exercise with regards to a neurally calculated safe exertion by the body and how brain could limit or improve physical performance (Noakes, 2012). Since then, several studies investigated and showed the essential role of the brain in the determination of fatigue and muscular strength and endurance performance (Gandevia, 2001; Noakes, 2011a,b, 2012). Thus, the development of innovative methods to aid in exercise performance is of great interest (Noakes, 2012; Van Cutsem et al., 2017a). One such method is transcranial direct current stimulation (tDCS).

tDCS is a noninvasive technique that emits a weak electrical current that can promote excitation, through tonic depolarization of the membrane resting potential (anodic stimulus, a-tDCS), or cortical inhibition, by hyperpolarization of the membrane resting potential (cathodic stimulus, c-tDCS) (Nitsche and Paulus, 2000; Stagg and Nitsche, 2011), i.e., increase or decrease of spontaneous firing rate of neurons affected by the electrical current (Bikson et al., 2004; Rahman et al., 2013).

Last years, several researchers have begun to verify the effects of tDCS on physical performance in healthy individuals (Lattari et al., 2016, 2017, 2018b; Angius et al., 2018). tDCS can be used as a neuromodulatory ergogenic resource for healthy individuals to change physical performance, such as lead to an increase in muscular strength (Lattari et al., 2016, 2017, 2018b), and endurance (Okano et al., 2015; Lattari et al., 2018a), in both nonathletes (Okano et al., 2015; Lattari et al., 2016, 2017, 2018a,b; Angius et al., 2018) and athletes (Sales et al., 2016; Hazime et al., 2017; Vargas et al., 2018) that have been using tDCS during their training programs (Reardon, 2016; Edwards et al., 2017). In line with this, the objective of this systematic review was to verify whether tDCS is an effective ergogenic resource for muscular strength and endurance in non-athletes and athletes.

\section{METHODS}

The method of this study was designed and reported according to the recommendations of the Preferred Reporting Items for Systematic reviews and Meta-Analyses (PRISMA) (Green and Higgins, 2011) and the Cochrane Handbook for Systematic Reviews of Interventions (Liberati et al., 2009).

Studies were included according to Participants, Intervention, Comparison, Outcomes, and Setting (PICOS) inclusion criteria. Participants were healthy men and women adults, athletes, strength and endurance training practitioners or sedentary, with no history of mioarticular injury and no psychiatric illness. Intervention were used the effects of the anode stimulus of tDCS (a-tDCS). Comparators were sham tDCS (i.e., the placebo stimulus) or no interventions (i.e., control). Outcomes for both the muscular strength and endurance were evaluated from different points of view: (1) physical tasks that consist of uniarticular exercise or multiarticular exercise; (2) physical performance that was measured objectively as endurance time, total work performed, force production during a maximal voluntary contraction (MVC), peak power, mean power, and/or time to exhaustion. All these variables are considered the primary outcomes of our review. Study Design were only randomized and non-randomized trials, using either cross-over or parallel group designs, comparing an intervention encompassing a-tDCS with a sham group on muscle strength or no intervention.

We analyzed only studies published in English language. A systematic literature search was conducted between December 10-2018 and January 10-2019. The following databases were used: PubMed, ISI Web of Science, and Scopus. No filters were applied in the search.

The search was performed using the terms physical exercise, strength training, resistance training, endurance training, cycling, effort, physical exertion, fatigue, and athletic performance, individually combined with transcranial direct current stimulation and tDCS, in all databases.

Included important reports and reviews regarding tDCS and muscle strength or endurance were manually screened for additional relevant studies. Experts on the field, including authors from the reports, were also requested to suggest any additional trials in order to ensure that the review was as comprehensive and up-to-date as possible.

To facilitate the interpretation of our results, the findings were structured in two categories: (i) studies on the effects of tDCS on muscle strength performance (ii) studies on the effects of tDCS on endurance performance. This strategy was used due to the need to differ these physical tasks in terms of physiological responses (Sidhu et al., 2013). In addition, after revision of the studies, future perspectives for new researches were proposed based on the gaps in the existing literature and ethical and regulatory issues related to the use of tDCS as an enhancer for physical performance in athletes.

\section{RESULTS}

The results identified a total of 1,067 articles (511 in the PubMed, 543 in Scopus, and 13 in Pedro). After the removal process of duplicate articles $(n=25)$, a total of 1,042 articles remained. One thousand sixteen articles were removed by title and/or abstract. After the removal process, 26 articles were included for systematic review, 18 examining the effects of tDCS on muscular strength performance and 8 on endurance performance. Flow chart is presented in Figure 1.

\section{tDCS for Improving Strength Performance}

A total of 18 studies (Cogiamanian et al., 2007; Tanaka et al., 2009; Kan et al., 2013; Williams et al., 2013; Hendy and Kidgell, 2014; Montenegro et al., 2015; Abdelmoula et al., 2016; Angius et al., 2016; Lattari et al., 2016, 2017, 2018b; Sales et al., 2016; Flood et al., 2017; Frazer et al., 2017; Hazime et al., 2017; Radel et al., 2017; Ciccone et al., 2018; Vargas et al., 2018) evaluated the efficiency of tDCS for improving muscular strength performance (see Tables 1, 2). No relevant side effects occurred.

\section{Study Characteristics}

In total, 496 participants, 282 males, and 214 females were included in the 19 studies. The mean age of the participants from studies varied between $16.01( \pm 0.9)$ (Vargas et al., 2018) and 27.7 ( \pm 8.4 ) (Kan et al., 2013) years. Regarding tDCS conditions, 


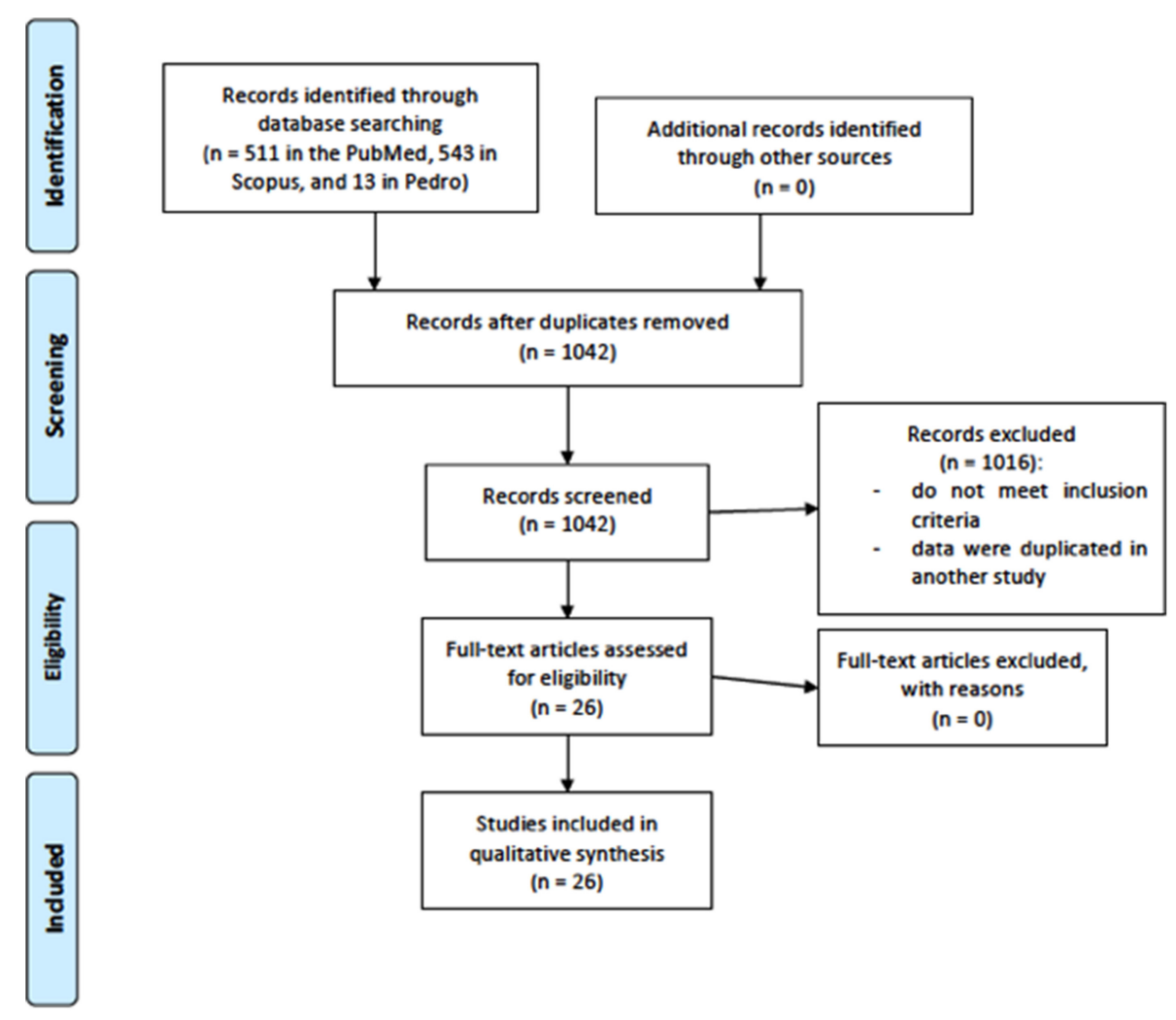

FIGURE 1 | Flow chart for study selection.

a-tDCS condition had sample sizes between 8 (Hazime et al., 2017) and 22 (Radel et al., 2017), with a total of 245 participants among studies, while control condition had sample sizes between 8 (Hazime et al., 2017) and 22 (Radel et al., 2017), with a total of 251 individuals among studies. Two studies had dropouts, $20 \%$ (2 participants) in the Tanaka's study (Tanaka et al., 2009) and 4.5\% (1 participant) in the Radel's study (Radel et al., 2017). As expected, most studies had more male than female participants. In addition, only five studies (Montenegro et al., 2015; Lattari et al., 2016, 2017, 2018b; Hazime et al., 2017) reported experience in ST, which could be an influencer factor in a-tDCS response. Regarding mode of tDCS application, twelve studies using offline mode (Cogiamanian et al., 2007; Kan et al., 2013; Williams et al., 2013; Montenegro et al., 2015; Abdelmoula et al., 2016; Angius et al., 2016; Lattari et al., 2016, 2017, 2018b; Flood et al., 2017; Frazer et al., 2017; Hazime et al., 2017), while six using online mode (Tanaka et al., 2009; Hendy and Kidgell, 2014; Sales et al., 2016; Radel et al., 2017; Ciccone et al., 2018; Vargas et al., 2018). Almost studies used crossover study design (Tanaka et al., 2009; Kan et al., 2013; Williams et al., 2013; Hendy and Kidgell, 2014; Montenegro et al., 2015; Abdelmoula et al., 2016; Angius et al., 2016; Lattari et al., 2016, 2017, 2018b; Sales et al., 2016; Flood et al., 2017; Frazer et al., 2017; Hazime et al., 2017; Radel et al., 2017; Ciccone et al., 2018; Vargas et al., 2018), and only one study integrated a parallel group study design (Cogiamanian et al., 2007), with only a single session of tDCS for both of them.

\section{Study Protocols for Muscular Strength Exercises}

All studies tested anodal tDCS in comparison to sham tDCS. Concerning tDCS conditions, a-tDCS protocol delivered stimulation on motor cortex (MC) (Cogiamanian et al., 2007; Tanaka et al., 2009; Kan et al., 2013; Williams et al., 2013; Hendy and Kidgell, 2014; Montenegro et al., 2015; Abdelmoula et al., 2016; Angius et al., 2016; Flood et al., 2017; Frazer et al., 2017; Hazime et al., 2017; Lattari et al., 2017; Radel et al., 2017; Ciccone et al., 2018; Vargas et al., 2018), dorsolateral prefrontal cortex (DLPFC) (Lattari et al., 2016, 2018b; Radel et al., 2017), and temporal cortex (TC) (Sales et al., 2016; Ciccone et al., 2018). Two studies used high-definition tDCS (Flood et al., 2017; Radel et al., 2017). The montage of electrodes respected a $4 \times 1$ ring configuration with the central electrode located over the hand cortical area (anodal) and return electrodes placed in a ring around the central anode (cathodal) at a radius around 5 and $4 \mathrm{~cm}$ (Flood et al., 2017; Radel et al., 2017). Electrodes with different sizes, i.e., between 12 and $35 \mathrm{~cm}^{2}$, were used in the target areas (Cogiamanian et al., 2007; Tanaka et al., 2009; Kan et al., 2013; Williams et al., 2013; Hendy and Kidgell, 2014; Montenegro et al., 2015; Abdelmoula et al., 2016; Angius et al., 2016; Lattari et al., 2016, 2017, 2018b; Sales et al., 2016; Frazer et al., 2017; Hazime et al., 2017; Ciccone et al., 2018; Vargas et al., 2018). In relation to the electrodes of $4 \mathrm{X} 1$ ring configuration, the estimated diameter was $1.1 \mathrm{~cm}$ (Flood et al., 2017; Radel et al., 


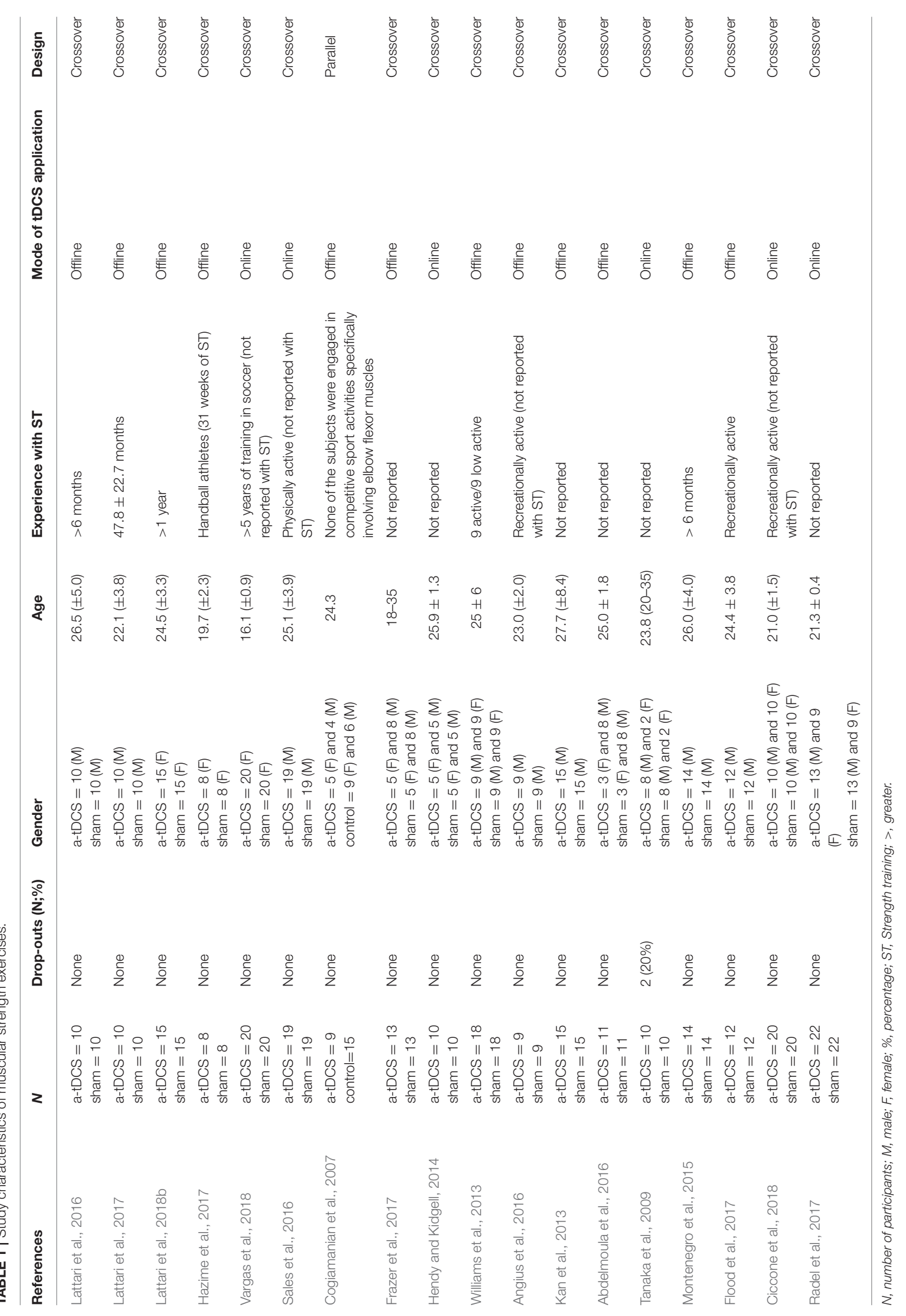


2017). Three studies applied an intensity of $1.5 \mathrm{~mA}$ (Cogiamanian et al., 2007; Williams et al., 2013; Abdelmoula et al., 2016) and the others used an intensity of $2 \mathrm{~mA}$ (Tanaka et al., 2009; Kan et al., 2013; Hendy and Kidgell, 2014; Montenegro et al., 2015; Angius et al., 2016; Lattari et al., 2016, 2017, 2018b; Sales et al., 2016; Flood et al., 2017; Frazer et al., 2017; Hazime et al., 2017; Radel et al., 2017; Ciccone et al., 2018; Vargas et al., 2018). In addition, session duration varied between 10 (Cogiamanian et al., 2007; Tanaka et al., 2009; Kan et al., 2013; Abdelmoula et al., 2016; Angius et al., 2016) and $20 \mathrm{~min}$ (Williams et al., 2013; Hendy and Kidgell, 2014; Montenegro et al., 2015; Lattari et al., 2016, 2017, 2018b; Sales et al., 2016; Flood et al., 2017; Frazer et al., 2017; Hazime et al., 2017; Radel et al., 2017; Ciccone et al., 2018; Vargas et al., 2018). With regard to control conditions, just one study applied no placebo stimulus (sham) (Cogiamanian et al., 2007) and the others used the sham condition (Tanaka et al., 2009; Kan et al., 2013; Williams et al., 2013; Hendy and Kidgell, 2014; Montenegro et al., 2015; Abdelmoula et al., 2016; Angius et al., 2016; Lattari et al., 2016, 2017, 2018b; Sales et al., 2016; Flood et al., 2017; Frazer et al., 2017; Hazime et al., 2017; Radel et al., 2017; Ciccone et al., 2018; Vargas et al., 2018). Fifteen studies utilized a $30 \mathrm{~s}$ period as sham stimulus (Tanaka et al., 2009; Kan et al., 2013; Williams et al., 2013; Hendy and Kidgell, 2014; Montenegro et al., 2015; Angius et al., 2016; Lattari et al., 2016, 2017, 2018b; Sales et al., 2016; Frazer et al., 2017; Hazime et al., 2017; Radel et al., 2017; Ciccone et al., 2018; Vargas et al., 2018) and three used other types of sham/control condition (Cogiamanian et al., 2007; Abdelmoula et al., 2016; Flood et al., 2017). The montage of the electrodes was the same as the a-tDCS condition.

The muscular strength exercise characteristics demonstrated that isometric (Cogiamanian et al., 2007; Tanaka et al., 2009; Kan et al., 2013; Abdelmoula et al., 2016; Angius et al., 2016; Flood et al., 2017; Hazime et al., 2017; Radel et al., 2017; Vargas et al., 2018) and dynamic contractions (Hendy and Kidgell, 2014; Montenegro et al., 2015; Lattari et al., 2016, 2017, 2018b; Sales et al., 2016; Frazer et al., 2017; Ciccone et al., 2018) were investigated. In dynamic exercises several types of tests were used, such as isokinetic testing (Montenegro et al., 2015; Sales et al., 2016; Ciccone et al., 2018), contractions against constant load (Hendy and Kidgell, 2014; Lattari et al., 2016, 2018b; Frazer et al., 2017), and muscular power (Lattari et al., 2017). Studies investigated the following muscles: elbow flexors (Cogiamanian et al., 2007; Kan et al., 2013; Williams et al., 2013; Abdelmoula et al., 2016; Lattari et al., 2016; Flood et al., 2017; Radel et al., 2017), internal and external rotator (Hazime et al., 2017), knee extensors (Montenegro et al., 2015; Angius et al., 2016; Sales et al., 2016; Lattari et al., 2017, 2018b; Ciccone et al., 2018; Vargas et al., 2018), adduction between the left great toe and the digitus secundus and adduction between the index finger and the thumb pad of the left hand (Tanaka et al., 2009), ankle, hip, and knee extensors (Lattari et al., 2017, 2018b), knee extensors and flexors (Montenegro et al., 2015). Alterations in muscular strength were examined through muscular endurance (Cogiamanian et al., 2007; Kan et al., 2013; Williams et al., 2013; Montenegro et al., 2015; Abdelmoula et al., 2016; Angius et al., 2016; Lattari et al., 2016, 2018b; Sales et al., 2016; Flood et al.,
2017; Radel et al., 2017; Ciccone et al., 2018), and maximal strength tests (Cogiamanian et al., 2007; Tanaka et al., 2009; Kan et al., 2013; Williams et al., 2013; Hendy and Kidgell, 2014; Abdelmoula et al., 2016; Angius et al., 2016; Flood et al., 2017; Frazer et al., 2017; Hazime et al., 2017; Vargas et al., 2018). Just one study examined the effects of a single session of tDCS on the muscular power (Lattari et al., 2017).

\section{The Effectiveness of tDCS on Improving the Muscular Strength Performance}

Regarding maximal voluntry contraction (MVC), two studies showed difference between a-tDCS and sham conditions (Hendy and Kidgell, 2014; Frazer et al., 2017). Both studies observed an increase in strength of untrained limbs. When analyzed the maximal isometric voluntary contractions (MIVC), no difference was observed between a-tDCS and sham conditions in 4 studies (Cogiamanian et al., 2007; Kan et al., 2013; Abdelmoula et al., 2016; Flood et al., 2017). Three studies showed a-tDCS was superior to sham condition in increase MIVC (Tanaka et al., 2009; Hazime et al., 2017; Vargas et al., 2018). The first in shoulder internal and external rotators of the shoulder (Hazime et al., 2017), the second in knee extensors (Vargas et al., 2018) and the third in the adduction between the left great toe and the digitus secundus (Tanaka et al., 2009). Regarding muscular endurance, there were finding significant differences between a-tDCS and sham conditions in seven studies (Cogiamanian et al., 2007; Williams et al., 2013; Abdelmoula et al., 2016; Angius et al., 2016; Lattari et al., 2016, 2018b; Sales et al., 2016). These differences were observed in isometric contraction (Cogiamanian et al., 2007; Williams et al., 2013; Abdelmoula et al., 2016; Angius et al., 2016), muscle action against a constant load (Lattari et al., 2016, 2018b) and isokinetic (Sales et al., 2016) strength tests. Six studies do not reveal significant differences between a-tDCS and sham conditions for muscular endurance in isometric contraction (Kan et al., 2013; Angius et al., 2016; Flood et al., 2017; Radel et al., 2017), and isokinetic (Montenegro et al., 2015; Ciccone et al., 2018) strength tests. For muscle power, one study was performed and showed that there was no significant difference between a-tDCS and sham condition (Lattari et al., 2017).

\section{tDCS for Improving Endurance Performance}

Eight studies (Angius et al., 2015, 2018; Okano et al., 2015; VitorCosta et al., 2015; Barwood et al., 2016; Sasada et al., 2017; Lattari et al., 2018a; Holgado et al., 2019) evaluated the efficiency of tDCS for improving the endurance (see Tables 3, 4). Relevant side effects were not described.

\section{Study Characteristics}

In total, 280 participants, 240 males and 40 females were included in the 8 studies. The mean age of the participants from studies varied between 21 (Sasada et al., 2017) and 33( \pm 9 ) (Okano et al., 2015) years. Regarding tDCS conditions, a-tDCS condition had sample sizes between 6 (Barwood et al., 2016) and 36 (Holgado et al., 2019), with a total of 133 participants among studies, while control condition had sample sizes between 6 (Barwood et al., 2016) and 36 (Holgado et al., 2019), with a total of 147 


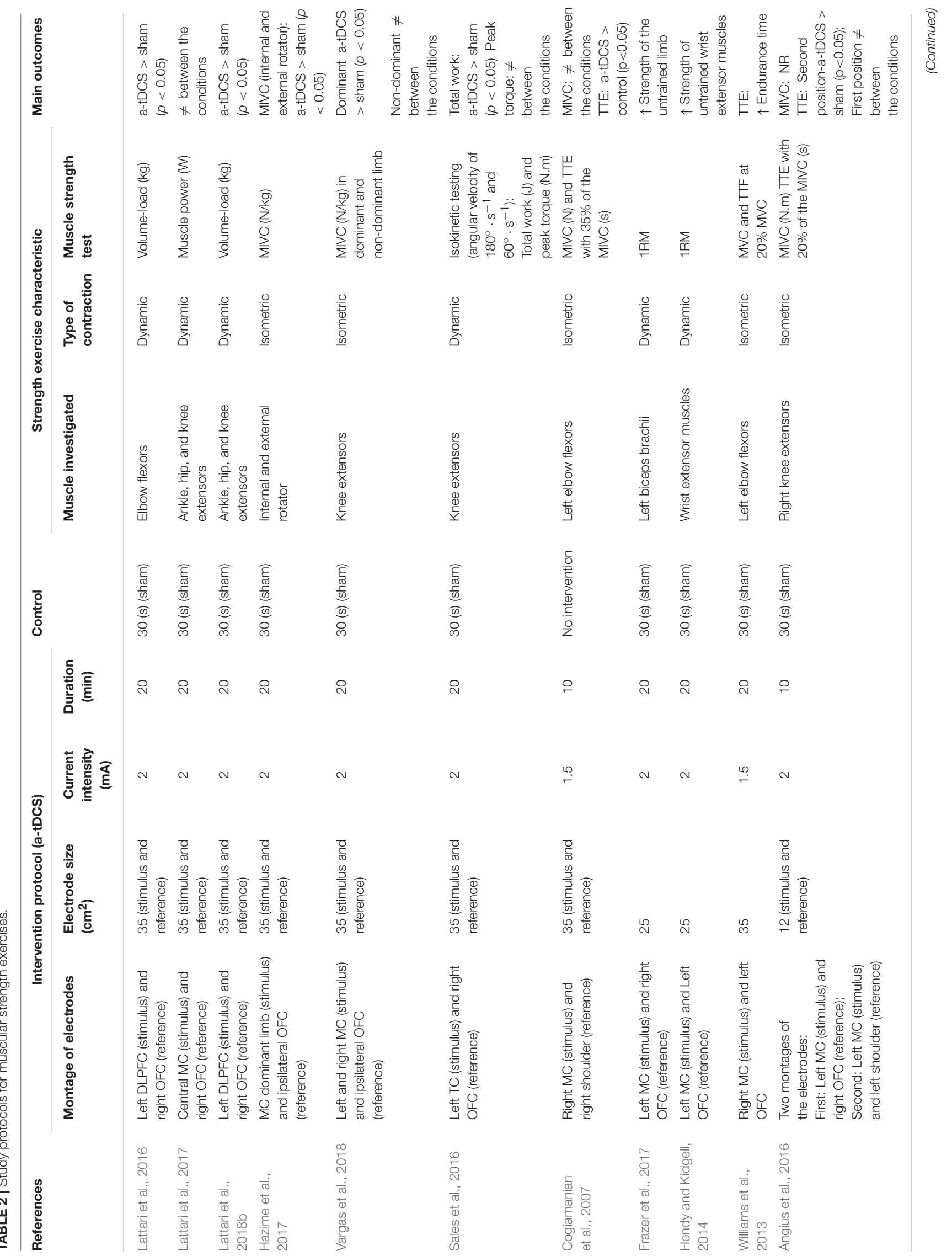




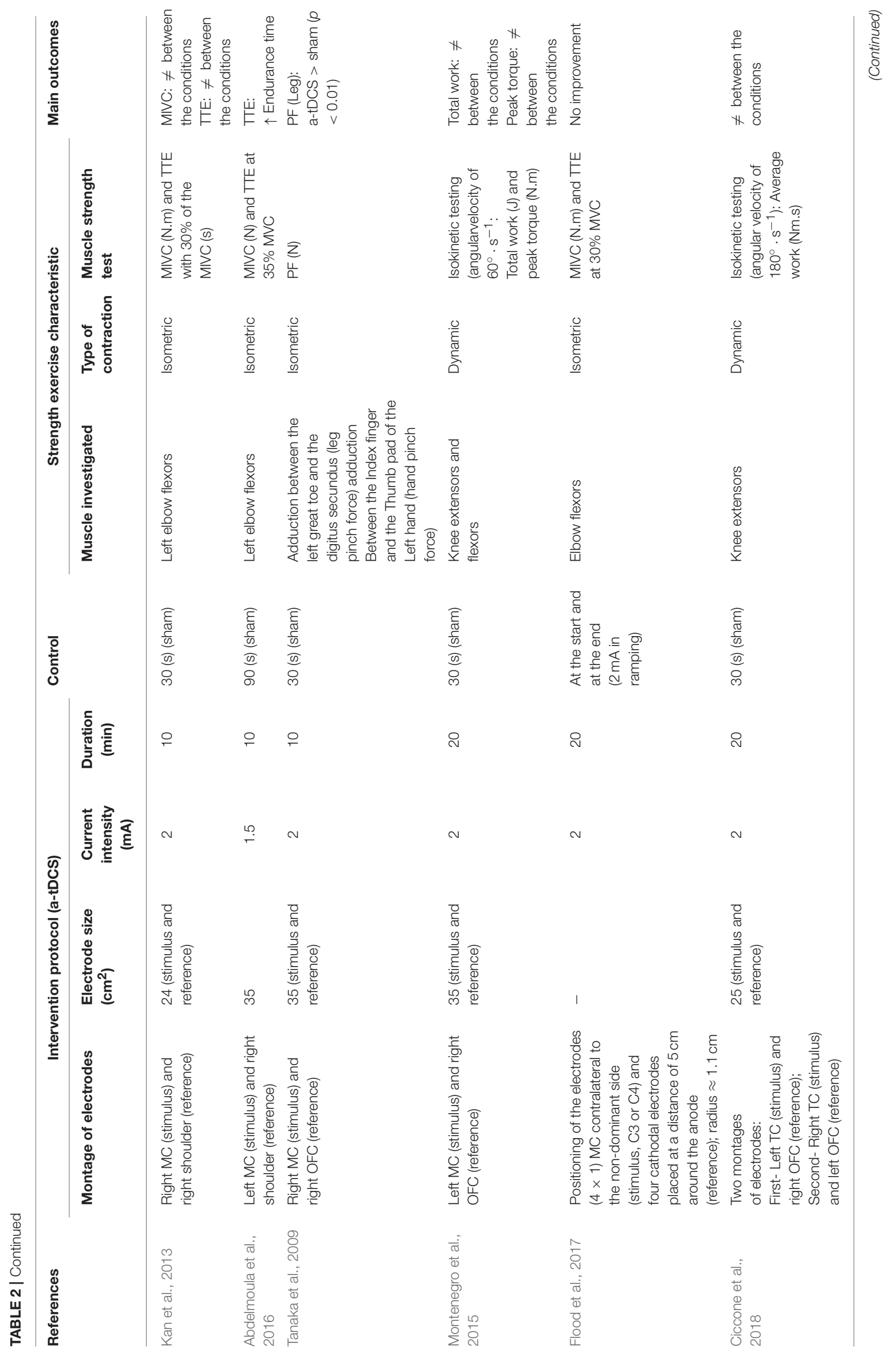




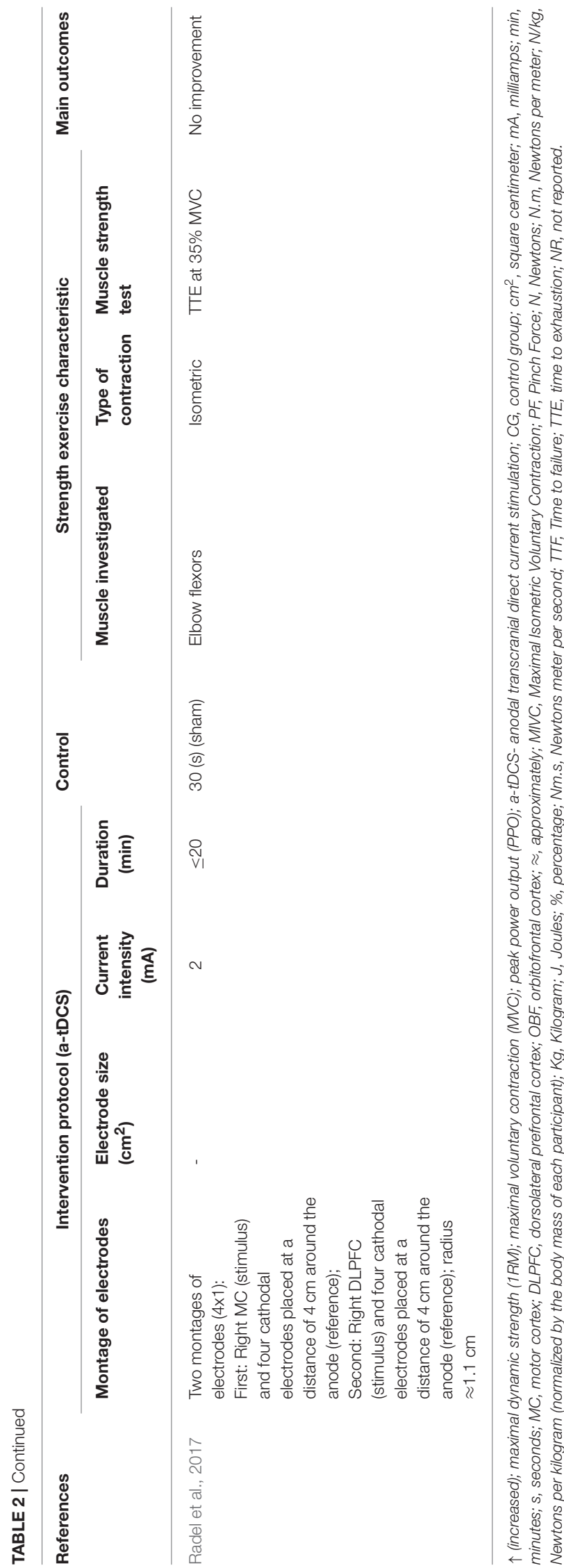

individuals among studies. There was no dropout. As expected, most studies had more female than male participants. In addition, only two studies (Okano et al., 2015; Sasada et al., 2017) reported experience in ET, which could be an influencer factor in a-tDCS response. Regarding mode of tDCS application, all studies using offline mode (Angius et al., 2015, 2018; Okano et al., 2015; VitorCosta et al., 2015; Barwood et al., 2016; Sasada et al., 2017; Lattari et al., 2018a; Holgado et al., 2019). In addition, all studies used crossover study design (Angius et al., 2015, 2018; Okano et al., 2015; Vitor-Costa et al., 2015; Barwood et al., 2016; Sasada et al., 2017; Lattari et al., 2018a; Holgado et al., 2019), with only a single session of tDCS for all of them.

\section{Study Protocols for Endurance Exercises}

All studies tested anodal tDCS in comparison to sham tDCS. Concerning tDCS conditions, a-tDCS protocol delivered stimulation on motor cortex (MC) (Angius et al., 2015, 2018; Vitor-Costa et al., 2015; Sasada et al., 2017), dorsolateral prefrontal cortex (DLPFC) (Lattari et al., 2018a; Holgado et al., 2019), and temporal cortex (TC) (Okano et al., 2015; Barwood et al., 2016). Electrodes with different sizes, i.e., between 12 and 36 $\mathrm{cm}^{2}$, were used in the target areas. All studies applied an intensity of 2 mA (Angius et al., 2015, 2018; Okano et al., 2015; Vitor-Costa et al., 2015; Barwood et al., 2016; Sasada et al., 2017; Lattari et al., 2018a; Holgado et al., 2019). In addition, two studies had session duration of 10 (Angius et al., 2015, 2018), one of 13 (Vitor-Costa et al., 2015), one of 15 (Sasada et al., 2017), and four of $20 \mathrm{~min}$ (Okano et al., 2015; Barwood et al., 2016; Lattari et al., 2018a; Holgado et al., 2019). With regard to control conditions, just one study applied no placebo stimulus plus sham (Angius et al., 2015) and the others used just the sham condition (Okano et al., 2015; Vitor-Costa et al., 2015; Barwood et al., 2016; Sasada et al., 2017; Angius et al., 2018; Lattari et al., 2018a; Holgado et al., 2019). All studies utilized a $30 \mathrm{~s}$ period as sham stimulus (Angius et al., 2015, 2018; Okano et al., 2015; Vitor-Costa et al., 2015; Barwood et al., 2016; Sasada et al., 2017; Lattari et al., 2018a; Holgado et al., 2019). The montage of the electrodes was the same as the a-tDCS condition.

The endurance exercise characteristics demonstrated that only cycling exercises (Angius et al., 2015, 2018; Okano et al., 2015; Vitor-Costa et al., 2015; Barwood et al., 2016; Sasada et al., 2017; Lattari et al., 2018a; Holgado et al., 2019) were used, and studied just lower limbs (Angius et al., 2015, 2018; Okano et al., 2015; Vitor-Costa et al., 2015; Barwood et al., 2016; Sasada et al., 2017; Lattari et al., 2018a; Holgado et al., 2019). The changes in endurance were investigated through maximal incremental test (Okano et al., 2015), $30 \mathrm{~s}$ maximal-effort sprint cycling test (Sasada et al., 2017), time-trial (TT) (Barwood et al., 2016; Holgado et al., 2019), time to exhaustion (TTE) (Angius et al., 2015; Vitor-Costa et al., 2015; Barwood et al., 2016; Lattari et al., 2018a), time to fatigue (TTF) (Angius et al., 2018) assessments.

\section{The Effectiveness of tDCS on Improving the Endurance Performance}

Regarding maximal incremental test, just one study investigated the effects of a-tDCS on endurance performance (Okano et al., 2015), and showed significant difference between a-tDCS and 


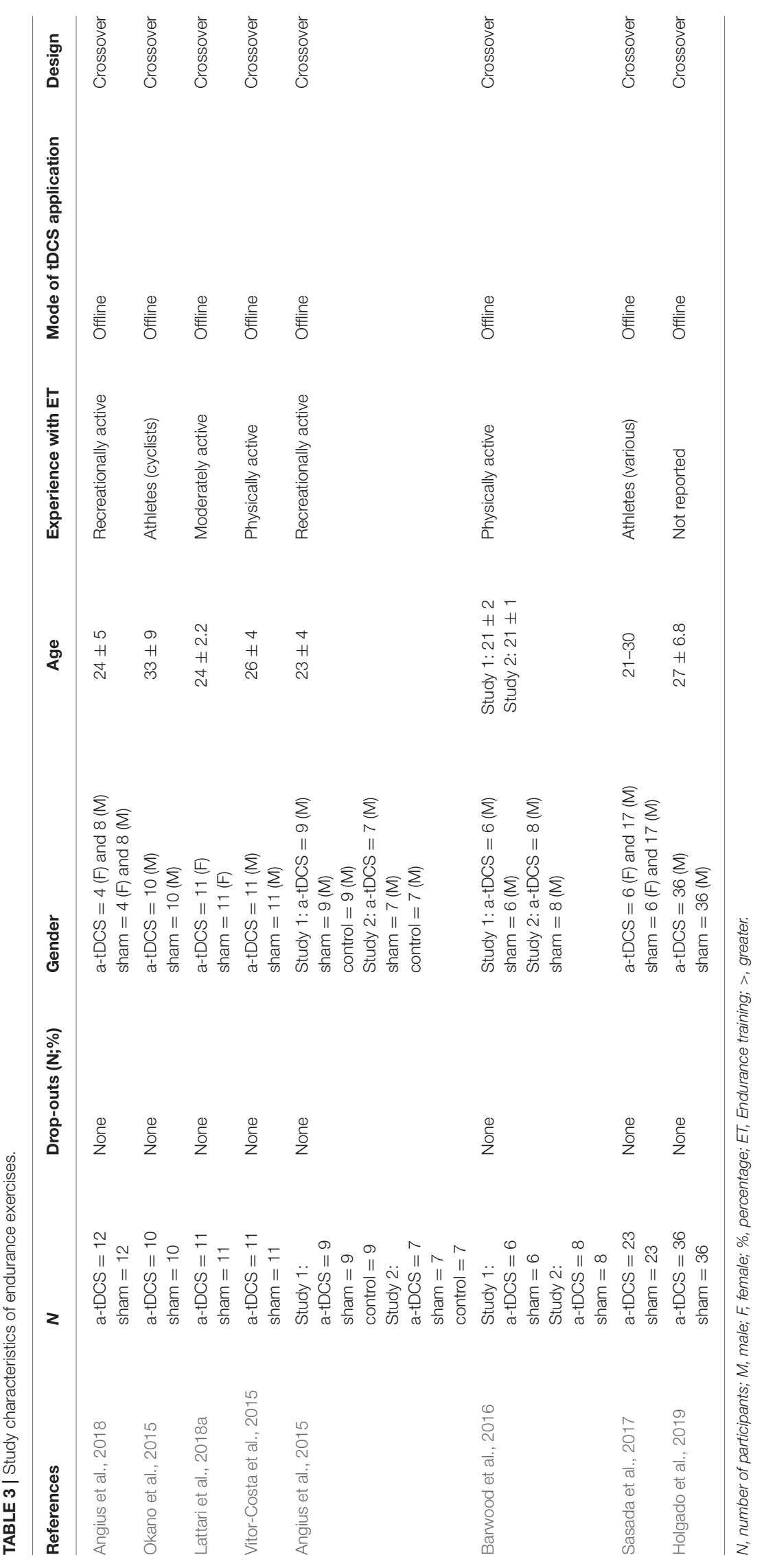




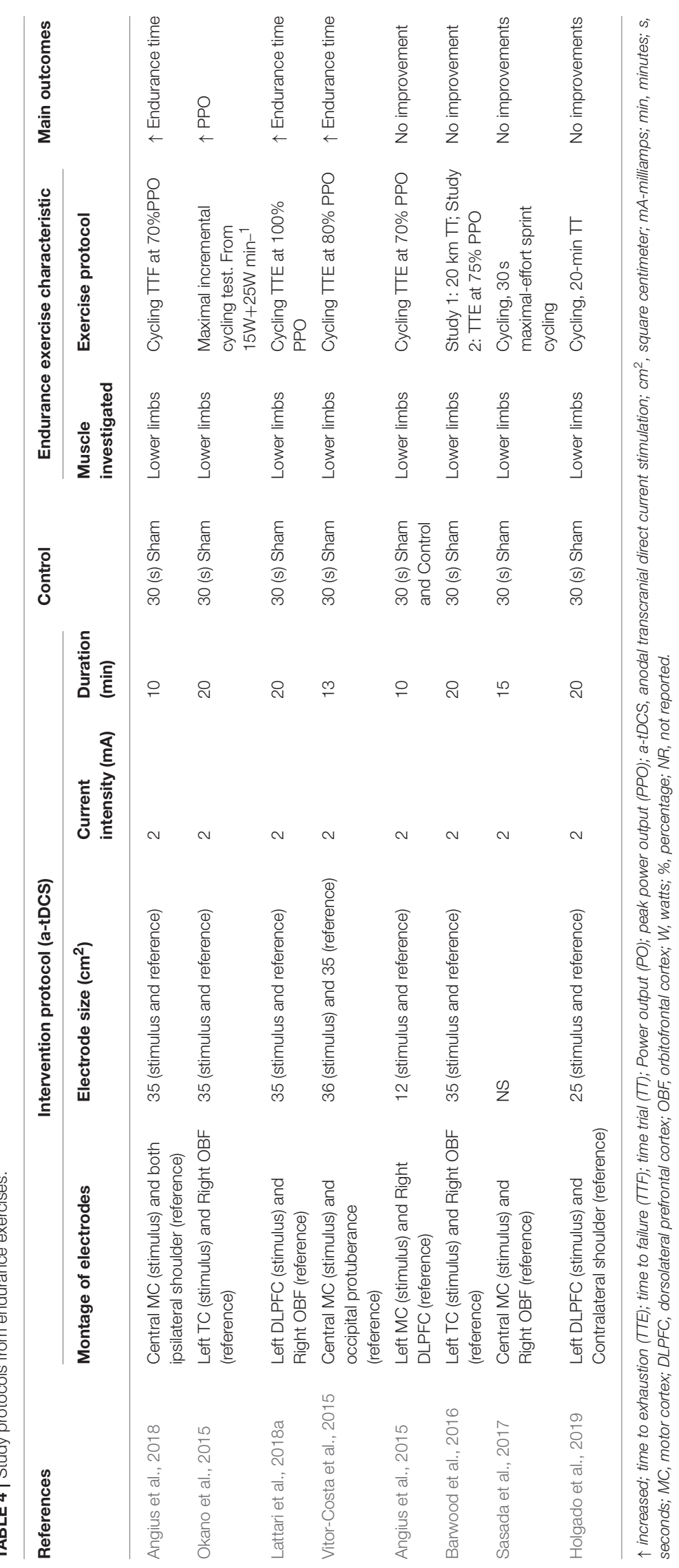


sham condition, with an increase in peak power output (PPO) after a-tDCS. Another study examined the effects of a-tDCS on $30 \mathrm{~s}$ maximal-effort sprint cycling test, revealed no significant difference between a-tDCS and sham condition (Sasada et al., 2017). Four articles examined the effects of a-tDCS on TTE (Angius et al., 2015; Vitor-Costa et al., 2015; Barwood et al., 2016; Lattari et al., 2018a). Two studies showed significant differences between a-tDCS and sham (Vitor-Costa et al., 2015; Lattari et al., 2018a), with increase in endurance time after a-tDCS, while two study did not reveal significant difference (Angius et al., 2015; Barwood et al., 2016). Two studies investigated the effects of atDCS on TT (Barwood et al., 2016; Holgado et al., 2019), with no study demonstrating significant difference between a-tDCS and sham condition. In addition, one study evaluated the effects of a-tDCS on TTF, with significant difference between a-tDCS and sham conditions. The authors showed an increase in endurance time after a-tDCS (Angius et al., 2018).

\section{DISCUSSION}

This review aimed to discuss the potential effects of tDCS as an ergogenic resource for muscular strength and endurance performance. The data of 26 controlled trials were analyzed (see Tables 1, 2). No trial mentions negative side effects of the intervention. The data show differences between the studies investigating muscle strength and the studies evaluating endurance, with regard to successful use of tDCS. Studies investigating the efficiency of tDCS on improving muscular strength demonstrate positive effects of a-tDCS in $66.7 \%$ of parameters tested. In contrast, in studies evaluating the effects of a-tDCS on improving endurance performance the a-tDCS revealed a significant improvement in only $50 \%$ of parameters assessed. The majority of the data shows consistently no influence of a-tDCS on muscular strength, but not to endurance performance. We will also discuss the potential directions of futures studies.

Due to the complex process which is the exercise practice, several brain areas may be involved in exercise regulation/limitation, and thus, a justification for the use of tDCS for performance improvement. However, most studies on tDCS and exercise performance and sports are not clear with respect to their hypotheses of why applying tDCS in a particular area of the brain for improving performance, such as the primary motor cortex (M1), the dorsolateral prefrontal cortex (CPFDL), and the insular cortex (IC).

Regarding brain areas, M1 is the most related to exercise performance due to its role in motor execution. Studies have consistently shown that central fatigue can compromise the physical performance of exercises of small muscle groups (e.g., elbow flexion), as well as exercises of large muscle groups (e.g., cycling). Specifically, spinal and supraspinal factors, such as reduced excitability of the motorneuron pool and the inability or limited ability of $\mathrm{M} 1$ and other supraspinal areas to increase the neural drive to compensate for this decrease in spinal excitability leads to decreased muscle capacity to produce strength/power and thus cause fatigue (Gandevia, 2001; Taylor and Gandevia, 2008; Taylor et al., 2016). Therefore, a reason to use tDCS over M1 would increase the excitability of it, which could result in sustained neural activity to the motor neuron, delay in the decrease of the neural unit to the active muscle and thus improve performance. In addition, other possible reasons for the application of tDCS over M1 could be modulate the pain perception. However, this mechanism still is unclear. A possible reason to direct M1 to pain modulation would be due to its connections with the insula and thalamus, as demonstrated in animal studies (Stepniewska et al., 1994). In addition, the atDCS in M1 increases the sensory and pain thresholds in healthy individuals as well as the level of pain in chronic pain patients (Vaseghi et al., 2014). In this regarding, it is suggested that exercise-induced pain plays a fundamental role in the regulation of performance, where individuals with better ability to tolerate or overcome pain would be more successful (Mauger, 2013). Therefore, the application of tDCS in M1 can also improve performance through exercise-induced pain attenuation.

With regard to PFC, whose main function is the cognitive control of behavior, seems to play an important role in processing internal and external cues related to the exercise performed (Robertson and Marino, 2016). PFC exerts a top-down influence that can result in changes of rhythm to complete the task, with prolongation of the motor output, slowing up the end of the exercise or the shutdown of the motor units, causing the end of the exercise (Robertson and Marino, 2016). Thus, the psychobiological model proposes this task of disengagement (that is, the end of the exercise) as a decision-making process based on the effort that depends on the motivation (for example, the maximal effort that a person is willing to exercise), perception of effort, knowledge of the endpoint of the exercise and distance/time remaining, and previous experience/memory of effort perception during exercise varying intensity and duration (Pageaux, 2014). A systematic review has confirmed that interventions aimed at decreasing the ability of PFC to exert control over bodily signals during exercise, such as mental fatigue (e.g., performing a cognitively prolonged task) may reduce endurance performance (Van Cutsem et al., 2017b). In fact, what has been observed is that there is a decrease in PFC oxygenation before the initiation of fatigue (Rupp and Perrey, 2008; Rooks et al., 2010). Therefore, the application of tDCS in the PFC could strengthen the ability of this region to disregard interoceptive cues (i.e., body signals), keeping the volitional drive to M1 and thus delaying the disengagement of the task (i.e., at the end of the exercise).

Another target area of tDCS studies on physical performance is the insular cortex (IC), considered as a responsible for cardiac autonomic control. Several types of studies indicate that the right IC is responsible for sympathetic modulation while the left IC is responsible for the parasympathetic modulation (Oppenheimer et al., 1992; Napadow et al., 2008). IC is a deep brain area, and theoretically it is modulated by tDCS through common connections with the temporal cortex (TC). For example, computational modeling and experimental studies showed that tDCS applied to left TC modulated IC activity, resulting in increased parasympathetic modulation at rest and during exercise (Montenegro et al., 2011; Okano et al., 2015). Within this context, the parasympathic branch is the responsible for modulating cardiac autonomic control at rest and when exercise begins a progressive decrease in modulation is observed until its complete withdrawal. 
Concerning the different brain areas stimulated, studies on tDCS show opposite results and a high variability regarding the effects on muscular strength and endurance performance. The high inter-individual variability, i.e., responders vs. nonresponders, to tDCS would be a possible explanation to the variance in outcomes (López-Alonso et al., 2015). Other factors like the different electrode montages used (see Tables 1, 2) and stimulation parameters (see Tables 3, 4) also can have contributed to mixed result. Furthermore, due to differences in stimulation parameters, such as electrode size and position, even as the low focality of tDCS (Miranda et al., 2013), other brain areas beyond the target area could be affected by the electric current from tDCS, changing the results completely. Overall, tDCS seems to enhance muscular strength and endurance performances.

Although there are many differences in terms of experimental design and physical task performed, some common characteristics can be found: (i) primary motor cortex (M1) has been the most targeted area; (ii) a-tDCS was delivered main before the physical task; (iii) most of the studies applied $20 \mathrm{~min}$ of stimulation at $2 \mathrm{~mA}$ with an active electrode size of $35 \mathrm{~cm}^{2}$. In relation to neuromuscular parameters, a-tDCS generally increased corticospinal excitability (Cogiamanian et al., 2007; Williams et al., 2013; Hendy and Kidgell, 2014; Frazer et al., 2017). Physiological responses during exercise did not show consistent changes after a-tDCS. Notably, when perceptual responses were measured, the improvement in physical performance induced by CTEF was often associated with a lower perceived exertion (Williams et al., 2013; Okano et al., 2015; Angius et al., 2016, 2018; Lattari et al., 2018b) while muscle pain did not change. The neurophysiological mechanisms that support the effect of a-tDCS on improving physical capacity are still unclear.

With respect to resistance, Cogiamanian et al. (2007) suggested that a-tDCS could improve subjects' motivation, reduce muscular pain, and modulate muscle synergy. However, none of the proposed mechanisms and corresponding parameters were monitored. Other authors propose that the improvement in endurance performance after a-tDCS could be due to increased neural drive and a reduction in supraspinatus fatigue (Williams et al., 2013; Vitor-Costa et al., 2015). Other authors have suggested that a-tDCS could influence sensorimotor integration and associated cognitive demand without altering the motor command (Abdelmoula et al., 2016). Angius et al. $(2016,2018)$ proposed that, due to the increase in a-tDCS-induced corticospinal excitability, fewer excitatory stimuli for M1 were required to produce the same submaximal force or power. As perceived exertion seems to depend on excitatory inputs from the supplemental motor area (SMA) and other brain regions (de Morree et al., 2012; Zenon et al., 2015), a reduction in such inputs would result in a lower perception of effort. It should be noted, however, that two studies reported improvements in endurance performance without significant changes in corticospinal excitability (Abdelmoula et al., 2016; Angius et al., 2016). This is not surprising, since previous studies have demonstrated a considerable variability in corticospinal response after tDCS over the motor cortex (MC) (Wiethoff et al., 2014; Madhavan et al., 2016).
Studies that investigated the effects of tDCS on muscle strength indicate that performance improvement was achieved both by increased corticospinal excitability and by reduced shortinterval intracortical inhibition and increased cross-activation (Hendy and Kidgell, 2014; Frazer et al., 2017). Other studies suggest that the improvement in workload was obtained by the reduction in the perception of effort (Lattari et al., 2016, 2018b). These mechanisms behind the tDCS's ergogenic effect remain unclear and should be interpreted with caution, since none of these studies monitored brain activity during exercise following tDCS.

\section{Limitations and Future Directions}

According to the rapid increase in the tDCS studies and muscular strength and endurance performance, important methodological limitations need to be considered. The different methodological characteristics of the experiments imply caution in interpret results related to effectiveness of tDCS as ergogenic aid. The standardization of methodological variables such as montage of electrodes, current intensity, session duration and other details, is essential to provide interesting insights about the real effects of tDCS on exercise and sport performance.

In addition, the mechanisms responsible for the improvements in muscular strength and endurance performances are still unclear. In line with this, an interest question is what results in the transient improvement in muscular strength and endurance performance? It seems that the modulation of corticospinal excitability or other targeted brain areas following tDCS would be the responsible for that improvement. Nevertheless, few studies examined corticospinal or brain activity following or during tDCS. Other technicality of tDCS is the low spatial resolution of the induced electric field in the brain when compared to transcranial magnetic stimulation (TMS) (Wagner et al., 2007a,b; Miranda et al., 2013), which can affect the functioning of certain brain areas beyond the target areas. The small sample found in the studies is other important point that can increase the probability of false positive results (Button et al., 2013). Lastly, the lack of appropriate blinding methods in most studies (see Tables 3, 4) should also be considered, since unapproved blinding procedure can lead to unexpected and confounding psychological effects, making difficult the interpretation of the results (Kessler et al., 2012; Fonteneau et al., 2019).

\section{CONCLUSION}

The results of this systematic review suggest that a-tDCS can improve muscular strength, but not to endurance performance. Nevertheless, evidence is insufficient to guarantee its effectiveness. New studies are required to assess the long-term effects of tDCS application combined with exercise training, whether with athletes or nonathletes. Despite tDCS is still considered a new tool in exercise and sport performance, it seems to have potential to improve performance. In line with this, more rigorous and extensive experimental studies are needed in order to better understand possible side effects from either regular use or 
abuse. Other important point that is needed is doing more studies with larger samples, appropriate blinding methods and techniques to examine neurophysiological mechanisms of tDCS.

\section{AUTHOR CONTRIBUTIONS}

SM and PJ designed the study, acquired and analyzed the data, and wrote the first draft of the paper. VA and JV helped to design

\section{REFERENCES}

Abdelmoula, A., Baudry, S., and Duchateau, J. (2016). Anodal transcranial direct current stimulation enhances time to task failure of a submaximal contraction of elbow flexors without changing corticospinal excitability. Neuroscience 322, 94-103. doi: 10.1016/j.neuroscience.2016.02.025

Angius, L., Hopker, J. G., Marcora, S. M., and Mauger, A. R. (2015). The effect of transcranial direct current stimulation of the motor cortex on exercise-induced pain. Eur. J. Appl. Physiol. 115, 2311-2319. doi: 10.1007/s00421-015-3212-y

Angius, L., Mauger, A. R., Hopker, J., Pascual-Leone, A., Santarnecchi, E., and Marcora, S. M. (2018). Bilateral extracephalic transcranial direct current stimulation improves endurance performance in healthy individuals. Brain Stimul. 11, 108-117. doi: 10.1016/j.brs.2017.09.017

Angius, L., Pageaux, B., Hopker, J., Marcora, S. M., and Mauger, A. R. (2016). Transcranial direct current stimulation improves isometric time to exhaustion of the knee extensors. Neuroscience 339, 363-375. doi: 10.1016/j.neuroscience.2016.10.028

Barwood, M. J., Butterworth, J., Goodall, S., House, J. R., Laws, R., Nowicky, A., et al. (2016). The effects of direct current stimulation on exercise performance, pacing and perception in temperate and hot environments. Brain Stimul. 9, 842-849. doi: 10.1016/j.brs.2016.07.006

Bikson, M., Inoue, M., Akiyama, H., Deans, J. K., Fox, J. E., Miyakawa, H., et al. (2004). Effects of uniform extracellular DC electric fields on excitability in rat hippocampal slices in vitro. J Physiol. 557(Pt 1), 175-190. doi: 10.1113/jphysiol.2003.055772

Button, K. S., Ioannidis, J. P. A., Mokrysz, C., Nosek, B. A., Flint, J., Robinson, E. S. J., et al. (2013). Power failure: why small sample size undermines the reliability of neuroscience. Nat. Rev. Neurosci. 14, 365-376. doi: 10.1038/nrn3475

Ciccone, A. B., Deckert, J. A., Schlabs, C. R., Tilden, M. J., Herda, T. J., Gallagher, P. M., et al. (2018). Transcranial direct current stimulation of the temporal lobe does not affect high intensity work capacity. J. Strength Cond. Res. doi: 10.1519/JSC.0000000000002561. [Epub ahead of print].

Cogiamanian, F., Marceglia, S., Ardolino, G., Barbieri, S., and Priori, A. (2007). Improved isometric force endurance after transcranial direct current stimulation over the human motor cortical areas. Eur. J. Neurosci. 26, 242-249. doi: 10.1111/j.1460-9568.2007.05633.x

de Morree, H. M., Klein, C., and Marcora, S. M. (2012). Perception of effort reflects central motor command during movement execution. Psychophysiology 49, 1242-1253. doi: 10.1111/j.1469-8986.2012.01399.x

Edwards, D. J., Cortes, M., Wortman-Jutt, S., Putrino, D., Bikson, M., Thickbroom, G., et al. (2017). Transcranial direct current stimulation and sports performance. Front. Hum. Neurosci. 11:243. doi: 10.3389/fnhum.2017.00243

Flood, A., Waddington, G., Keegan, R. J., Thompson, K. G., and Cathcart, S. (2017). The effects of elevated pain inhibition on endurance exercise performance. PeerJ 5:e3028. doi: 10.7717/peerj.3028

Fonteneau, C., Mondino, M., Arns, M., Baeken, C., Bikson, M., Brunoni, A. R., et al. (2019). Sham tDCS: a hidden source of variability? Reflections for further blinded, controlled trials. Brain Stimul. 12, 668-673.

Frazer, A. K., Williams, J., Spittle, M., and Kidgell, D. J. (2017). Cross-education of muscular strength is facilitated by homeostatic plasticity. Eur. J. Appl. Physiol. 117, 665-677. doi: 10.1007/s00421-017-3538-8

Gandevia, S. C. (2001). Spinal and supraspinal factors in human muscle fatigue. Physiol. Rev. 81, 1725-1789. doi: 10.1152/physrev.2001.81.4.1725

Green, S., and Higgins, J. (eds). (2011). Cochrane Handbook for Systematic Reviews of Interventions. Chichester: John Wiley \& Sons, Ltd. the study, to organize the data acquired, and to discuss the first draft of the paper.

\section{FUNDING}

SM was supported by grant from Carlos Chagas Foundation for the Research Support in the State of Rio de Janeiro (FAPERJ), Young Scientists from the State of Rio de Janeiro, E-26/203.295/2017.

Hazime, F. A., da Cunha, R. A., Soliaman, R. R., Romancini, A. C. B., Pochini, A. D. C., Ejnisman, B., et al. (2017). Anodal transcranial direct current stimulation (TDCS) increases isometric strength of shoulder rotators muscles in handball players. Int. J. Sports Phys. Ther. 12, 402-407.

Hendy, A. M., and Kidgell, D. J. (2014). Anodal-tDCS applied during unilateral strength training increases strength and corticospinal excitability in the untrained homologous muscle. Exp. Brain Res. 232, 3243-3252. doi: $10.1007 / \mathrm{s} 00221-014-4016-8$

Holgado, D., Zandonai, T., Ciria, L. F., Zabala, M., Hopker, J., and Sanabria, D. (2019). Transcranial direct current stimulation (tDCS) over the left prefrontal cortex does not affect time-trial self-paced cycling performance: evidence from oscillatory brain activity and power output. PLOS ONE 14:e0210873. doi: 10.1371/journal.pone.0210873

Kan, B., Dundas, J. E., and Nosaka, K. (2013). Effect of transcranial direct current stimulation on elbow flexor maximal voluntary isometric strength and endurance. Appl. Physiol. Nutr. Metab. 38, 734-739. doi: 10.1139/apnm-2012-0412

Kessler, S. K., Turkeltaub, P. E., Benson, J. G., and Hamilton, R. H. (2012). Differences in the experience of active and sham transcranial direct current stimulation. Brain Stimul. 5, 155-162. doi: 10.1016/j.brs.2011.02.007

Lattari, E., Andrade, M. L., Filho, A. S., Moura, A. M., Neto, G. M., Silva, J. G., et al. (2016). Can transcranial direct current stimulation improve the resistance strength and decrease the rating perceived scale in recreational weight-training experience? J. Strength Cond. Res. 30, 3381-3387. doi: 10.1519/JSC.0000000000001457

Lattari, E., Campos, C., Lamego, M. K., Passos de Souza, S. L., Neto, G. M., Rocha, N. B., et al. (2017). Can transcranial direct current stimulation improve muscle power in individuals with advanced resistance training experience? J. Strength Cond. Res. doi: 10.1519/JSC.0000000000001956. [Epub ahead of print].

Lattari, E., de Oliveira, B. S., Oliveira, B. R. R., de Mello Pedreiro, R. C., Machado, S., and Neto, G. A. M. (2018a). Effects of transcranial direct current stimulation on time limit and ratings of perceived exertion in physically active women. Neurosci. Lett. 662, 12-16. doi: 10.1016/j.neulet.2017.10.007

Lattari, E., Rosa Filho, B. J., Fonseca Junior, S. J., Murillo-Rodriguez, E., Rocha, N., Machado, S., et al. (2018b). Effects on volume load and ratings of perceived exertion in individuals advanced weight-training after transcranial direct current stimulation. J. Strength Cond. Res. doi: 10.1519/JSC.0000000000002434. [Epub ahead of print].

Liberati, A., Altman, D. G., Tetzlaff, J., Mulrow, C., Gotzsche, P. C., Ioannidis, J. P. A., et al. (2009). The PRISMA statement for reporting systematic reviews and meta-analyses of studies that evaluate healthcare interventions: explanation and elaboration. BMJ 339:b2700. doi: 10.1136/bmj.b2700

López-Alonso, V., Ferna’ndez-Del-Olmo, M., Costantini, A., Gonzalez-Henriquez, J. J., and Cheeran, B. (2015). Intra-individual variability in the response to anodal transcranial direct current stimulation. Clin. Neurophysiol. 126, 2342-2347. doi: 10.1016/j.clinph.2015.03.022

Madhavan, S., Sriraman, A., and Freels, S. (2016). Reliability and variability of tDCS induced changes in the lower limb motor cortex. Brain Sci. 6:26. doi: $10.3390 /$ brainsci6030026

Mauger, A. R. (2013). Fatigue is a pain-the use of novel neurophysiological techniques to understand the fatigue-pain relationship. Front. Physiol. 4:104. doi: 10.3389/fphys.2013.00104

McCormick, A., Meijen, C., and Marcora, S. (2015). Psychological determinants of whole-body endurance performance. Sport Med. 45, 997-1015 doi: 10.1007/s40279-015-0319-6 
Miranda, P. C., Mekonnen, A., Salvador, R., and Ruffini, G. (2013). The electric field in the cortex during transcranial current stimulation. Neuroimage 70 , 48-58. doi: 10.1016/j.neuroimage.2012.12.034

Montenegro, R., Okano, A., Gurgel, J., Porto, F., Cunha, F., Massaferri, R., et al. (2015). Motor cortex tDCS does not improve strength performance in healthy subjects. Rev. Educ. Fis. 21, 185-193. doi: 10.1590/S1980-65742015000200009

Montenegro, R. A., Farinatti, P. T. V., Fontes, E. B., Soares, P. P. S., Cunha, F. A., Gurgel, J. L., et al. (2011). Transcranial direct current stimulation influences the cardiac autonomic nervous control. Neurosci. Lett. 497, 32-36. doi: 10.1016/j.neulet.2011.04.019

Napadow, V., Dhond, R., Conti, G., Makris, N., Brown, E. N., and Barbieri, R. (2008). Brain correlates of autonomic modulation: combining heart rate variability with fMRI. Neuroimage 42, 169-177. doi: 10.1016/j.neuroimage.2008.04.238

Neumayr, G., Hoertnagl, H., Pfister, R., Koller, A., Eibl, G., and Raas, E. (2003). Physical and physiological factors associated with success in professional alpine skiing. Int. J. Sport Med. 24, 571-575. doi: 10.1055/s-2003-43270

Nitsche, M. A., and Paulus, W. (2000). Excitability changes induced in the human motor cortex by weak transcranial direct current stimulation. J. Physiol. 527, 633-639. doi: 10.1111/j.1469-7793.2000.t01-1-00633.x

Noakes, T. D. (2011a). Time to move beyond a brainless exercise physiology: the evidence for complex regulation of human exercise performance. Appl. Physiol. Nutr. Metab. 36, 23-35. doi: 10.1139/H10-082

Noakes, T. D. (2011b). Is it time to retire the A.V. Hill Model?: a rebuttal to the article by Professor Roy Shephard. Sport Med. 41, 263-277. doi: 10.2165/11583950-000000000-00000

Noakes, T. D. (2012). Fatigue is a brain-derived emotion that regulates the exercise behavior to ensure the protection of whole body homeostasis. Front. Physiol. 3, 1-13. doi: 10.3389/fphys.2012.00082

Okano, A. H., Fontes, E. B., Montenegro, R. A., Farinatti, Pde, T., Cyrino, E. S., Noakes, T. D., et al. (2015). Brain stimulation modulates the autonomic nervous system, rating of perceived exertion and performance during maximal exercise. Br. J. Sports Med. 49, 1213-1218. doi: 10.1136/bjsports-2012091658

Oppenheimer, S. M., Gelb, A., Girvin, J. P., and Hachinski, V. C. (1992). Cardiovascular effects of human insular cortex stimulation. Neurology 42, 1727-1732. doi: 10.1212/WNL.42.9.1727

Pageaux, B. (2014). The psychobiological model of endurance performance: an effort-based decision-making theory to explain self-paced endurance performance. Sport Med. 2014:1-3. doi: 10.1007/s40279-014-0198-2

Radel, R., Tempest, G., Denis, G., Besson, P., and Zory, R. (2017). Extending the limits of force endurance: stimulation of the motor or the frontal cortex? Cortex 97, 96-108. doi: 10.1016/j.cortex.2017.09.026

Rahman, A., Reato, D., Arlotti, M., Gasca, F., Datta, A., Parra, L. C., et al. (2013). Cellular effects of acute direct current stimulation: somatic and synaptic terminal effects. J. Physiol. 591, 2563-2578. doi: 10.1113/jphysiol.2012.247171

Reardon, S. (2016). 'Brain doping' may improve athletes' performance. Nature 531, 283-284. doi: 10.1038/nature.2016.19534

Robertson, C. V., and Marino, F. E. (2016). A role for the prefrontal cortex in exercise tolerance and termination. J. Appl. Physiol. 120, 464-466. doi: 10.1152/japplphysiol.00363.2015

Rooks, C. R., Thom, N. J., McCully, K. K., and Dishman, R. K. (2010). Effects of incremental exercise on cerebral oxygenation measured by nearinfrared spectroscopy: a systematic review. Prog. Neurobiol. 92, 134-150. doi: $10.1016 /$ j.pneurobio.2010.06.002

Rupp, T., and Perrey, S. (2008). Prefrontal cortex oxygenation and neuromuscular responses to exhaustive exercise. Eur. J. Appl. Physiol. 102, 153-163. doi: 10.1007/s00421-007-0568-7

Sales, M. M., De Sousa, C. V., Browne, R. A. V., Fontes, E. B., Olher, R. R. V., Ernesto, C., et al. (2016). Transcranial direct current stimulation improves muscle isokinetic performance of young trained individuals. Med. Dello Sport $69,1-10$.

Sasada, S., Endoh, T., Ishii, T., and Komiyama, T. (2017). Polarity-dependent improvement of maximal-effort sprint cycling performance by direct currentstimulation of the central nervous system. Neurosci Lett. 657, 97-101. doi: 10.1016/j.neulet.2017.07.056

Savulescu, J., Foddy, B., and Clayton, M. (2004). Why we should allow performance enhancing drugs in sport. Br. J. Sports Med. 38, 666-670. doi: 10.1136/bjsm.2003.005249
Schubert, M. M., and Astorino, T. A. (2012). A systematic review of the efficacy of ergogenic aids for improving running performance. J. Strength Cond. Res. 27, 1699-1707. doi: 10.1519/JSC.0b013e31826cad24

Sidhu, S. K., Cresswell, A. G., and Carroll, T. J. (2013). Corticospinal responses to sustained locomotor exercises: moving beyond single-joint studies of central fatigue. Sports Med. 43, 437-449. doi: 10.1007/s40279-013-0020-6

Sleivert, G. G., and Rowlands, D. (1996). Physical and physiological factors associated with success in the triathlon. Sport Med. 22, 8-18. doi: 10.2165/00007256-199622010-00002

Stagg, C. J., and Nitsche, M. A. (2011). Physiological basis of transcranial direct current stimulation. Neuroscientist 17, 37-53. doi: 10.1177/1073858410386614

Stepniewska, I., Preuss, T. M., and Kaas, J. H. (1994). Thalamic connections of the primary motor cortex (M1) of owl monkeys. J. Comp. Neurol. 349, 558-582. doi: 10.1002/cne.903490405

Tanaka, S., Hanakawa, T., Honda, M., and Watanabe, K. (2009). Enhancement of pinch force in the lower leg by anodal transcranial direct current stimulation. Exp. Brain Res. 196, 459-465. doi: 10.1007/s00221-009-1863-9

Taylor, J. L., Amann, M., Duchateau, J., Meeusen, R., and Rice, C. L. (2016). Neural contributions 1 to muscle fatigue: from the brain to the muscle and back. Med. Sci. Sport Exerc. 48, 2294-2306. doi: 10.1249/MSS.0000000000000923

Taylor, J. L., and Gandevia, S. C. (2008). A comparison of central aspects of fatigue in submaximal and maximal voluntary contractions. J. Appl. Physiol. 104, 542-550. doi: 10.1152/japplphysiol.01053.2007

Van Cutsem, J., De Pauw, K., Buyse, L., Marcora, S., Meeusen, R., and Roelands, B. (2017a). Effects of mental fatigue on endurance performance in the heat. Med. Sci. Sports Exerc. 49, 1677-1687. doi: 10.1249/MSS.0000000000001263

Van Cutsem, J., Marcora, S., De Pauw, K., Bailey, S., Meeusen, R., and Roelands, B. (2017b). The effects of mental fatigue on physical performance: a systematic review. Sport Med. 47, 1569-1588. doi: 10.1007/s40279-016-0672-0

Vargas, V. Z., Baptista, A. F., Pereira, G. O. C., Pochini, A. C., Ejnisman, B., Santos, M. B., et al. (2018). Modulation of isometric quadriceps strength in soccer players with transcranial direct current stimulation: a crossover study. J. Strength Cond. Res. 32, 1336-1341. doi: 10.1519/JSC.0000000000001985

Vaseghi, B., Zoghi, M., and Jaberzadeh, S. (2014). Does anodal transcranial direct current stimulation modulate sensory perception and pain? A meta-analysis study. Clin. Neurophysiol. 125, 1847-1858. doi: 10.1016/j.clinph.2014.01.020

Vitor-Costa, M., Okuno, N. M., Bortolotti, H., Bertollo, M., Boggio, P. S., Fregni, F., et al. (2015). Improving cycling performance: transcranial direct current stimulation increases time to exhaustion in cycling. PLoS ONE 10:e0144916 doi: 10.1371/journal.pone.0144916

Wagner, T., Fregni, F., Fecteau, S., Grodzinsky, A., Zahn, M., and Pascual-Leone, A. (2007a). Transcranial direct current stimulation: a computer-based human model study. Neuroimage 35, 1113-1124. doi: 10.1016/j.neuroimage.2007.01.027

Wagner, T., Valero-Cabre, A., and Pascual-Leone, A. (2007b). Noninvasive human brain stimulation. Annu. Rev. Biomed. Eng. 9, 527-565. doi: 10.1146/annurev.bioeng.9.061206.133100

Wiethoff, S., Hamada, M., and Rothwell, J. C. (2014). Variability in response to transcranial direct current stimulation of the motor cortex. Brain Stimul. 7, 468-475. doi: 10.1016/j.brs.2014.02.003

Williams, P. S., Hoffman, R. L., and Clark, B. C. (2013). Preliminary evidence that anodal transcranial direct current stimulation enhances time to task failure of a sustained submaximal contraction. PLOS ONE 8:e81418. doi: 10.1371/journal.pone.0081418

Zenon, A., Sidib_e, M., and Olivier, E. (2015). Disrupting the supplementary motor area makes physical effort appear less effortful. J. Neurosci. 35, 8737-8744. doi: 10.1523/JNEUROSCI.3789-14.2015

Conflict of Interest Statement: The authors declare that the research was conducted in the absence of any commercial or financial relationships that could be construed as a potential conflict of interest.

Copyright (c) 2019 Machado, Jansen, Almeida and Veldema. This is an open-access article distributed under the terms of the Creative Commons Attribution License (CC $B Y)$. The use, distribution or reproduction in other forums is permitted, provided the original author(s) and the copyright owner(s) are credited and that the original publication in this journal is cited, in accordance with accepted academic practice. No use, distribution or reproduction is permitted which does not comply with these terms. 